\title{
Plasmonic Sensors for Disease Detection - A Review
}

\section{Barizuddin $\mathbf{S}^{2}$, Bok $\mathbf{S}^{2}$ and Gangopadhyay $\mathbf{S}^{1,2 *}$}

${ }^{1}$ Department of Electrical and Computer Engineering, University of Missouri, Columbia, USA

${ }^{2}$ Center for Microsystems and Nanotechnology, University of Missouri, Columbia, USA

\begin{abstract}
Surface Plasmon Resonance (SPR) and Localized Surface Plasmon Resonance (LSPR) have become powerful bio-sensing techniques. These techniques allow for rapid and ultra-sensitive detection of biological analytes with applications in medical diagnostics, environmental monitoring, and food safety. Plasmonic sensors are fabricated using metal nanoparticles, metal nanostructures or a combination of both. With major advancements in the area of nano synthesis, the development of plasmonic sensors has expanded significantly. Plasmonic platforms offer a viable substitute to detection techniques such as Enzyme Linked Immunosorbent Assay (ELISA) and Polymerase Chain Reaction (PCR) in some cases and complement them in many others. Most of the state-of-the-art plasmonic biosensors can measure binding affinity and kinetic rates in real time from any molecule that can change its refractive index. Other developments in plasmonics include the use of metamaterials that operate in the visible and infra-red region. In this review, we will briefly cover the developments in the last few years in plasmonics as it relates to disease detection.
\end{abstract}

Keywords: Plasmonics; Bio-sensing; Nano; Diagnostics; Optical sensing

\section{Introduction}

Conventional optics are limited in their ability to resolve nanometer-scale structures because of the diffraction limit [1-3]. This is because the evanescent waves carrying the sub-wavelength information decay before they form an image when they pass through a medium of different permittivity [3-7]. To overcome this limitation, researchers have worked to develop methods for sub-wavelength imaging [8-11]. Although some methods like immersion lenses and near-field imaging were able to detect some sub-wavelength features, they could not form distinct images. The development in the field of plasmonics has enabled the detection of sub-wavelength features by compensating the evanescent loss and restoring the image below the diffraction limit.

The development of plasmonics has led to extraordinary advancement in the area of ultra-sensitive detection at the single particle or molecular level. New developments in nano-synthesis have made it possible to design nanoparticles in different sizes, shapes and configurations [12-17]. Noble metal nanoparticles of gold $(\mathrm{Au})$ and silver $(\mathrm{Ag})$ exhibit excellent plasmonic properties and have widely been used for sensing low-level and label-free analytes in physiological media [18]. The plasmonic sensing is determined by the resonance shift of a plasmonic sensor in the presence of the bio-analytes which changes the refractive index of dielectrics around the metal. This unique property of the plasmonic resonance comes from the plasmon oscillation or the cumulative motion of the conduction band electrons relative to fixed positive ions that are often observed in noble metals [19-21]. When the plasmonic oscillations are induced by outside light sources in specific wavelength ranges and specific angles, they can confine and control the light at the nanoscale [22].

The noble metals exhibit very different properties when they are on a nanoscale. When light is incident on the nanoparticles it makes the conduction electrons in them oscillate with a resonant frequency [23-26]. These oscillations are a function of nanoparticle shape, size and morphology. As a result of these LSPR modes [2630], the nanoparticles absorb and scatter light very intensely. These novel characteristics enable the nanoparticles to be used as labels for immunoassays in bio-sensors [31-34]. Other applications of plasmonic nanoparticles [35,36] include their utility as optical switches [37,38], waveguides [39,40] and lithographic tools [41-44]. Though LSPR phenomenon is theoretically possible in any metal, silver and gold are the most commonly used metals [45-47]. Gold as a material is favored for plasmonic sensors due to its corrosion resistance and its binding capability to biomolecules. However, silver appears to be the material of choice due to the low dielectric loss at different optical frequencies. Other common materials that exhibit plasmonic properties include aluminum and copper [48-50]. Plasmonic nanoparticles also serve as spatial labels $[51,52]$ in immunoassays and cellular imaging and act as transducers $[28,53,54]$ that convert small changes in the refractive index into spectral shifts. The refractive index of the organic biomolecules is different from the buffer. The binding of the nanoparticles with the biomolecules has the effect of an increased refractive index that results in the spectrum shifting to a higher wavelength (red-shift) $[46,55,56]$. These bio-molecular interactions can be monitored in real time with high sensitivity [57]. LSPR-shift assays are also useful for the ultrasensitive quantification of proteins [58]. In this review, our intent is to present briefly the development of plasmonic sensors in the last few years as it relates to the diagnosis of disease.

\section{Detection of Biomarkers}

\section{Detection of thrombin for blood clotting}

Sensing platforms using SPR can measure modest changes in the refractive index emanating from the interactions between two bioentities close to the surface of the plasmonic "element". The binding kinetics near the surface can be measured in real time [59-61]. While aggregation-based immunoassays use simple instrumentation, biosensor sensitivity is limited by the number of aggregation events and require a long incubation period to ensure maximized signals. As a result, sensing platforms using SPR spectroscopy are used as signal transduction methods for measuring the changes in refractive index

*Corresponding authors: Shubhra Gangopadhyay, 349 Engineering Building West University of Missouri, Columbia, MO 65211, USA, Tel: 5738829266; Fax: 5738840312; E-mail: gangopadhyays@missouri.edu

Received April 28, 2016; Accepted May 03, 2016; Published May 10, 2016

Citation: Barizuddin S, Bok S, Gangopadhyay S (2016) Plasmonic Sensors for Disease Detection - A Review. J Nanomed Nanotechnol 7: 373. doi:10.4172/21577439.1000373

Copyright: (C 2016 Barizuddin S, et al. This is an open-access article distributed under the terms of the Creative Commons Attribution License, which permits unrestricted use, distribution, and reproduction in any medium, provided the original author and source are credited. 
Citation: Barizuddin S, Bok S, Gangopadhyay S (2016) Plasmonic Sensors for Disease Detection - A Review. J Nanomed Nanotechnol 7: 373. doi:10.4172/2157-7439.1000373

that result from analyte binding to the recognition element at the surface of the noble metal thin film or nanoparticle. Many SPR sensors [4852] have recently been used for such applications. A similar technique was used for the detection of thrombin utilizing thrombin aptamer functionalized gold $\mathrm{Au}$ ) aggregated nanoparticles which were used as seeds for the growth in the presence of CTAB, HAuCl4 and NADH [6267]. For further amplification, the thrombin aptamer was immobilized on a glass surface and incubated with different concentrations of thrombin. The aptamer functionalized $\mathrm{Au}$ nanoparticles were bound to the second available site on the thrombin as shown in Figure $1 \mathrm{~A}$ with concentrations of thrombin ranging between 0 and $160 \mathrm{nM}$ as shown in the absorbance spectra (Figure 1B). A wavelength of $650 \mathrm{~nm}$ was used to generate the calibration curve as shown in Figure 1C.

\section{Detection of prostate specific antigen (PSA) for prostate cancer}

Elliptical nano-disk arrays were fabricated using thermal Nanoimprint lithography (NIL) with the enzyme-antibody conjugate to enhance PSA detection. The Au nano-disks were fabricated as shown in Figure 2. The morphology of the optically anisotropic elliptical Au nano-disk arrays was studied by SEM, as shown in Figure 2A and 2B. The long-axis and short axis of the Au nano-disk named Ii and Is show different extinction peaks (Figure 2C).
The Au nano-disks modified with 11-mercaptoundecanoic acid (MUA) are shown in Figure 3A. The functionalized nano-disks were incubated in a solution of anti-PSA, to immobilize the antibody (Figure 3B). After that the PSA, biotinylated anti-PSA, and streptavidin-alkaline phosphatase were applied to form the sandwich assay (Figure 3C and 3D). Finally, the enzyme-catalyzed precipitation reaction (shown in Figure $3 \mathrm{E}$ and $3 \mathrm{~F}$ ) was shown to yield insoluble precipitates onto the nano-disk surfaces. Without the enzymatic precipitation, PSA detection in the 2.8 $\mathrm{nM}$ range was considered to be difficult, but with enzymatic precipitation, a concentration of $280 \mathrm{fM}$ was detected with peak shifts of 7.7 and 5.5 in the s-peak and I-peak respectively [53]. Enzyme-linked immunosorbent assay (ELISA) using LSPR can be viewed physically with the eye, without any equipment. In conventional ELISA, the intensity of the color is a function of the concentration of the target molecule that impedes the visible detection in equivalent concentration ranges.

Detection of carcinoembryonic antigen (CEA) for colon cancer and prostate specific antigen for prostate cancer

Blood contains protein biomarkers that are the indicators of certain diseases. However, the ultra-low concentrations of biomarkers secreted by colon and prostate tumors in early stages are not high enough to show clinical symptoms. Nano-plasmonic biosensors can
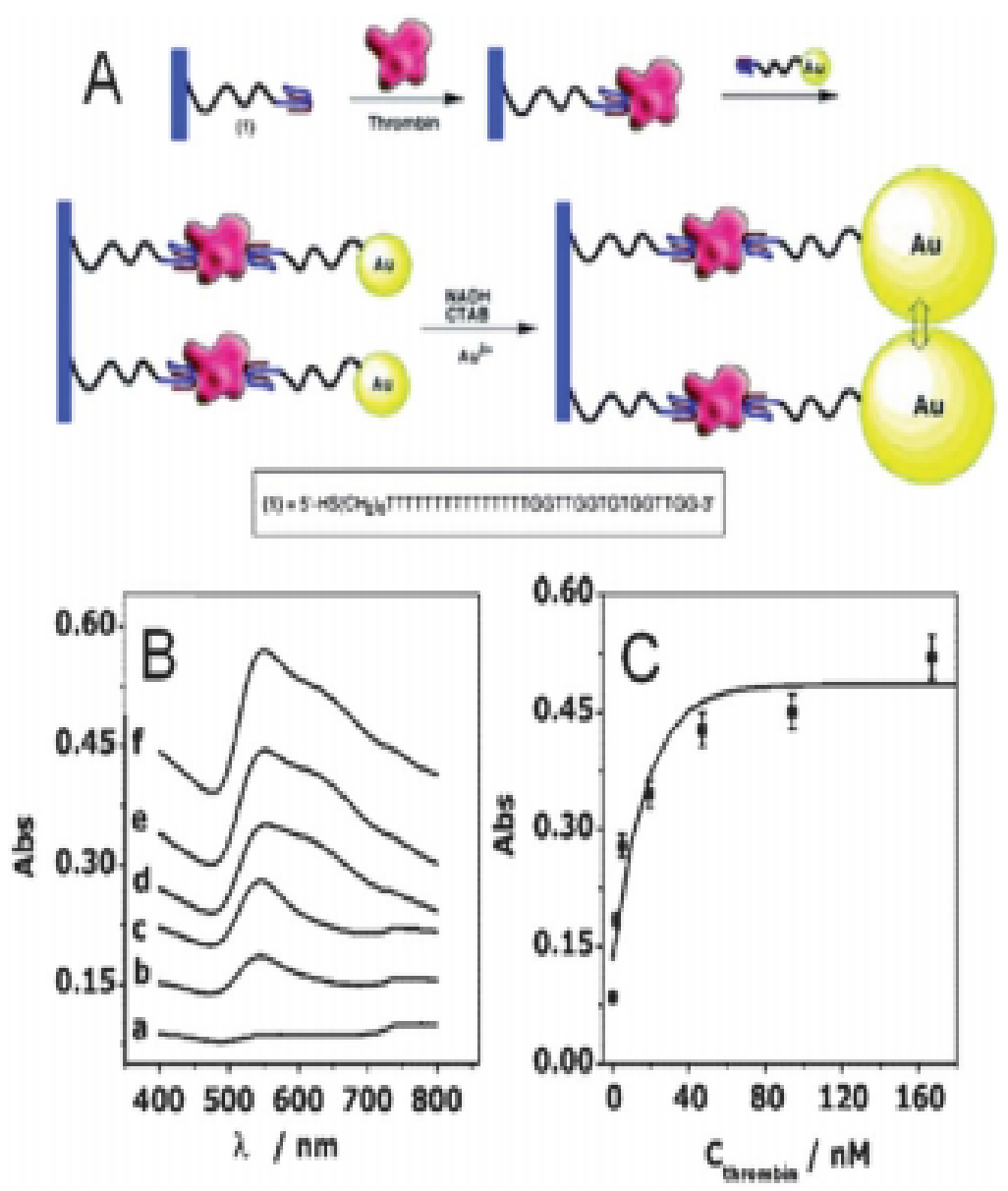

Figure 1: (A) Amplified detection of thrombin on surfaces by the catalytic enlargement of thrombin aptamer-functionalized gold nanoparticles. (B) Absorbance spectra of thiolated aptamer modified glass slides incubated in (a) 0 (b) 2 (c) 5 (d) 19 (e) 94 and (f) 167 nM thrombin using thiolated aptamer functionalized gold nanoparticles and the catalytic enlargement process. (C) Calibration curve corresponding to the amplified optical detection of thrombin (Reprinted with permission from, Copyright 2014 American Chemical Society [67]). 
Citation: Barizuddin S, Bok S, Gangopadhyay S (2016) Plasmonic Sensors for Disease Detection - A Review. J Nanomed Nanotechnol 7: 373. doi:10.4172/2157-7439.1000373
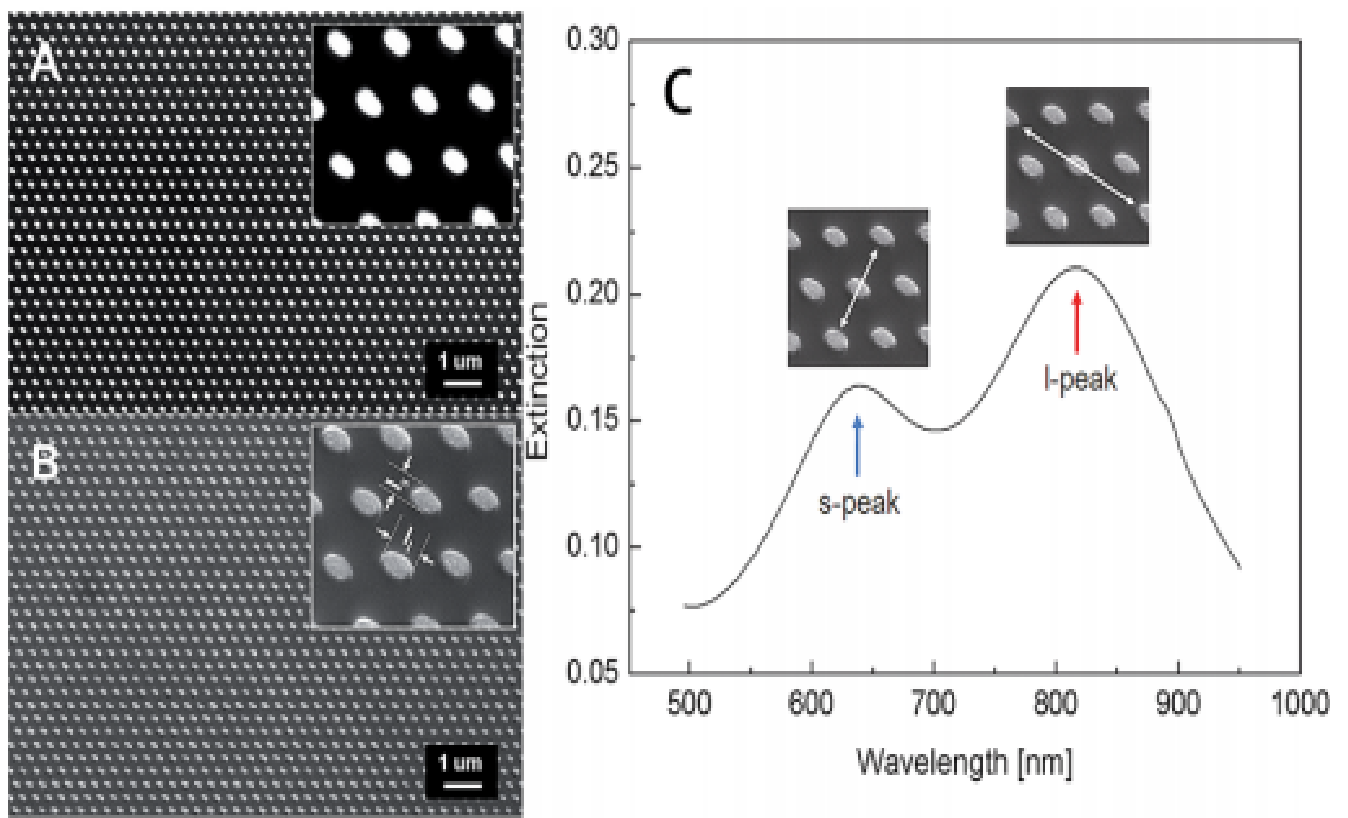

Figure 2: SEM images of (A) the array pattern of the elliptical Si nanopillars, (B) the array pattern of elliptical Au nanodisks was fabricated on a glass wafer (C) as prepared elliptical Au nano-disk arrays, including s-peak and l-peak. (Reproduced with permission, from Copyright @ 2011 American Chemical Society [53]).

(a) Au elliptical nanodisk

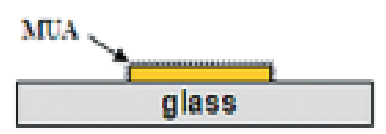

(d)

\section{Enzyme-antibody \\ conjugate}

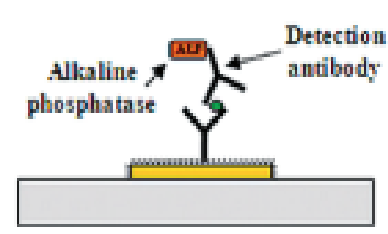

(b) Capture antibody
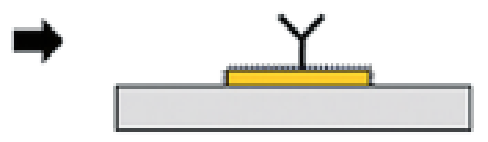

(e)
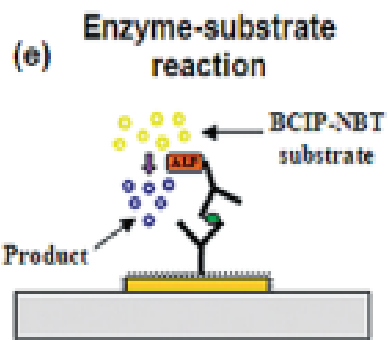

(c) PSA antigen
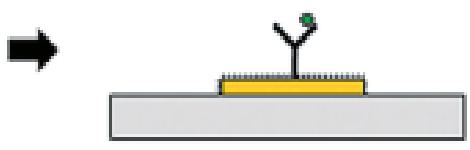

Product

(f) precipitation

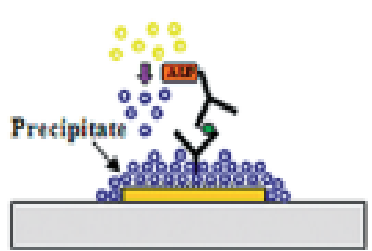

Figure 3: Schematic illustration of PSA detection procedure, (A) Modification of the surface MUA. (B) Immobilization of the PSA antibody. (C) Binding of PSA to the antibody. (D) Sandwich binding of alkaline phosphatase detection antibody conjugates to PSA. (E) Enzymatic reaction of the BCIP/NBT substrate. (F) Product precipitation on the nano-disk. (Reproduced with permission from Copyright @ 2011 American Chemical Society. This journal is $($ ) The Royal Society of Chemistry 2014 RSC Adv., 2014, 4, 43725-43745|43735 Review RSC Advances Published on 04 September 2014 [53]).

provide exceptional levels of sensitivity for detecting disease states. The sandwich assay in Figure 4 shows such a system in which a cancer biomarker using cantilever is primarily recognized by surface-anchored antibodies and then by an antibody in a solution that identifies a region of the captured biomarker [68]. The second antibody is bound to a $100 \mathrm{~nm}$ diameter gold nanoparticle. The nanoparticle has a mass of $\sim 10 \mathrm{fg}$ and a density of $19,300 \mathrm{~kg} \mathrm{~m}^{-3}$ [68]. It also exhibits localized plasmonic resonance in the visible range which results in resonant enhancement of light absorption and scattering. The micro cantilevers also display a structural quality that can be used as optical resonators. The bio-molecular interactions [69-75] on the cantilever results in the optical spectral shift as explained earlier. Carcinoembryonic Antigen and prostate specific antigen (PSA) were detected by analyzing these samples spiked in undiluted serum. This technique improved the LOD by three orders of magnitude compared to the other clinical diagnostic systems. This detection method for CEA and PSA involves two biorecognition steps to enhance the selectivity and improve the sensor response as indicated in Figure 4. The resonant frequency is a function of the loading of biomolecules on the cantilever. The mass loading indicates a decrease in the resonance frequency. The intrinsic detection limit for these devices in the air was about $1 \mathrm{pg}$ (10-12 g), which was corroborated by the approximate number of detected molecules, which was found to be about 100 . 
Citation: Barizuddin S, Bok S, Gangopadhyay S (2016) Plasmonic Sensors for Disease Detection - A Review. J Nanomed Nanotechnol 7: 373. doi:10.4172/2157-7439.1000373

a

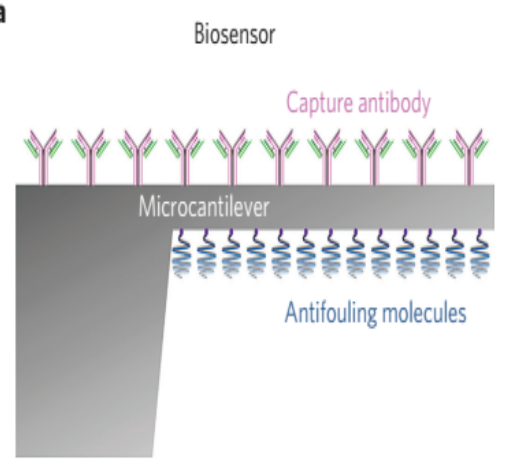

Biomarker capture

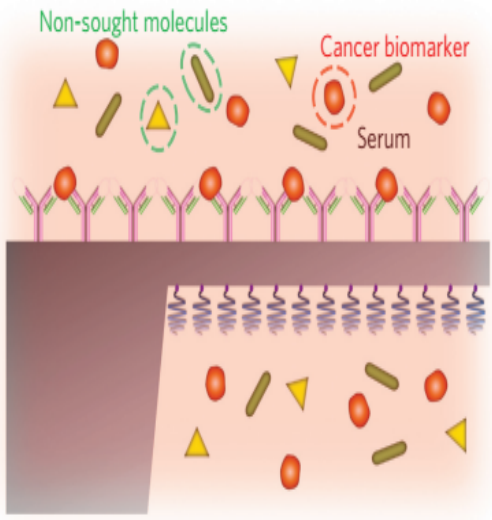

Sandwich assay

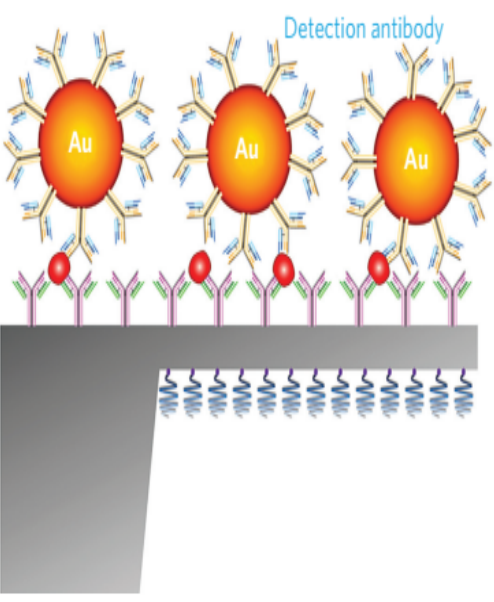

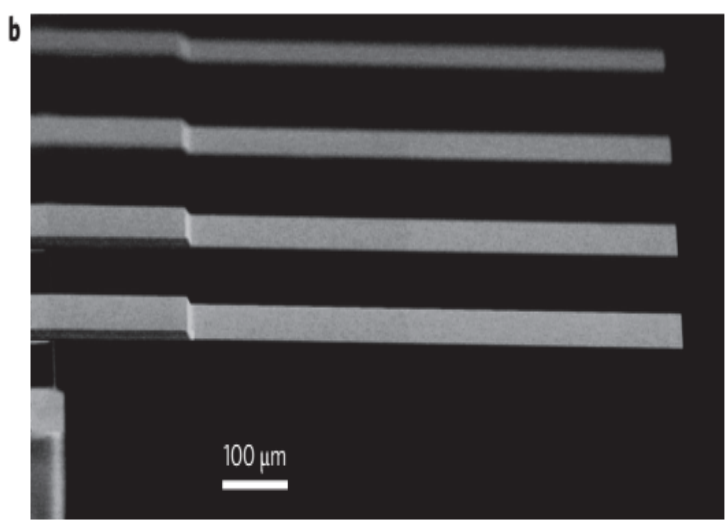

C

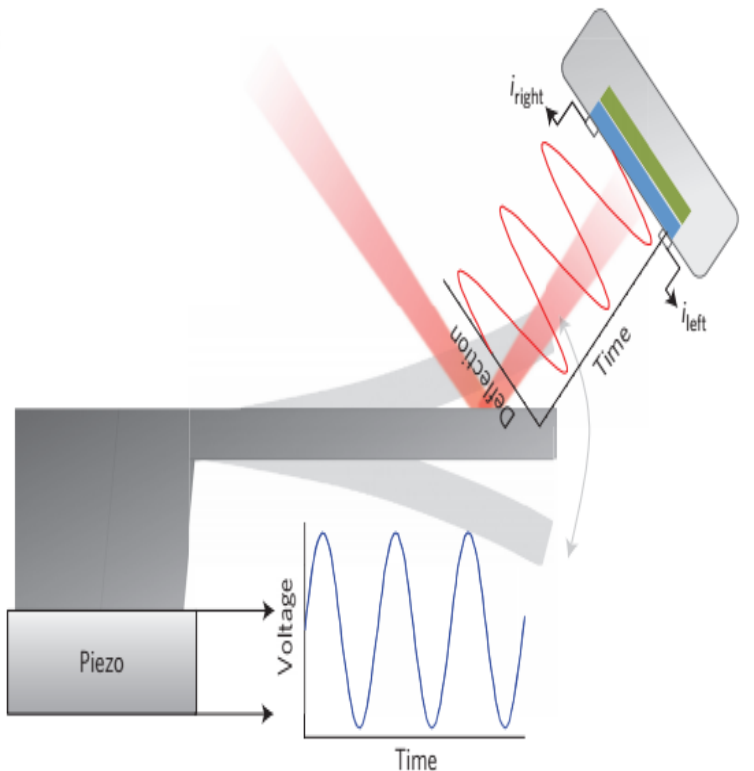

d

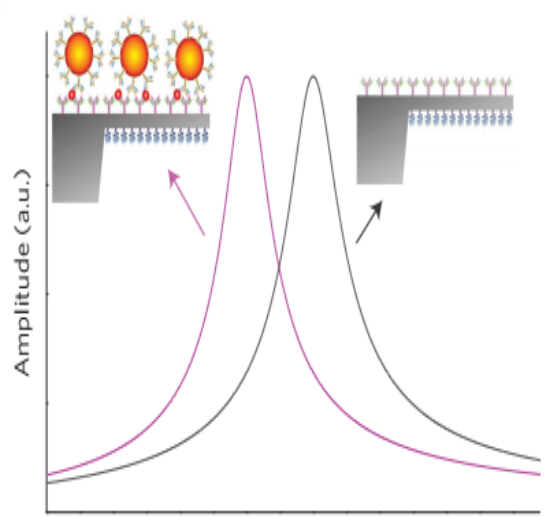

Frequency (a.u.)

Figure 4: Schematic illustration of the sandwich assay on the cantilevers and its effect on the resonance frequency. (A) The cantilever was functionalized with capture antibodies. The functionalization method comprised of silanization, followed by antibody binding on the top surface of the cantilever. Polyethylene glycol was used to minimize nonspecific interactions. The cantilever was then immersed in the serum sample for immunoreaction to take place between the protein biomarker and the capture antibodies. The immunoreactions were apparent on exposing the cantilever to the detection antibodies for the biomarker that was bound to 100-nm-diameter gold nanoparticles. The detection antibody recognizes a specific open free region of the captured biomarker. (B) SEM image of the silicon cantilevers. The cantilever dimensions were $500 \mu \mathrm{m}$ long, $100 \mu \mathrm{m}$ wide and $1 \mu \mathrm{m}$ thick (C) Optical beam deflection method for measuring the vibrations of the cantilever in which a laser beam was focused on the free-end region. The deflection of the reflected beam by the cantilever vibrations was measured by a linear position-sensitive photodetector. The photocurrents I-right and I-left were measured by the linear position-sensitive photodetector. The cantilever array was driven by a piezoelectric actuator located beneath the base of the cantilever. (D) Nanoparticle mass loading effect on the resonance frequency of the cantilever. The resulting downshift of the resonance frequency represents the proportion to the added mass. (Reproduced with permission from Ref. [68]. Copyright @ Nature Publishing Group). 
Citation: Barizuddin S, Bok S, Gangopadhyay S (2016) Plasmonic Sensors for Disease Detection - A Review. J Nanomed Nanotechnol 7: 373. doi:10.4172/2157-7439.1000373

Page 5 of 10

The opto-plasmonic transduction method used to capture biomarkers is shown in Figure 5, in which there is a light scattering of the gold nanoparticles as a result of the LSPR. The nanoparticles spectra of the light scattered due to the presence of a single biofunctionalized nanoparticle on the substrate indicates a peak at a wavelength of 620 which gives the orange color of the nanoparticle [76]. The binding of the gold nanoparticle to the silicon surface breaks the degeneration of the dipole plasmonic modes [77,78], which results in different optical frequencies based on the orientation. The opposite sides of the cantilever act as mirrors which enhances the light reflectivity by useful interference between the reflection from the two surfaces. This property was used for detecting ultralow concentrations of the cancer biomarker.

\section{Detection of squamous cell carcinoma antigen (SCCa) for cervical cancer}

Squamous cell carcinoma antigen (SCCa) is a tumor biomarker that is used for the diagnosis, treatment and prognosis for patients suffering from cervical cancer [79]. A LSPR technique based on noble metal nanoparticles bypasses the disadvantages of traditional testing strategies, it improves free-labeling, has short assay time, excellent sensitivity, and selectivity $[27,28,64,72,80,81]$. A LSPR biosensor for the detection of SCCa is shown in Figure 6 in which triangle-shaped Ag nanoparticle array was fabricated using nano-sphere lithography using 11-mercaptoundecanoic acid (MUA) to form a functionalized chip surface with monoclonal anti-SCCa antibodies. Different concentrations of SCCa were effectively tested in both buffer and human serum with detections in the linear range of 0.1-1,000 pM. The sensor functionalization was a multi-step process as outlined in Figure 6. The LSPR spectra for the detection of SCCa shown in Figure 7 indicates a total red-shift of 24.95 from the bare substrate to the immobilized 100 pM SCCa.

\section{Detection of surface antigen (HBsAg) for hepatitis B}

Plasmon-enabled diagnostic assays are mostly performed in solution [82]. The advantage of using plasmonics is that being in nano-scale, they have a large surface area. The size of the particles and their high diffusion rates in the sample allow for increased speed and sensitivity compared to surface-based approaches. Assays, where the analyte molecule is directly confined on the nanoparticle surface tend

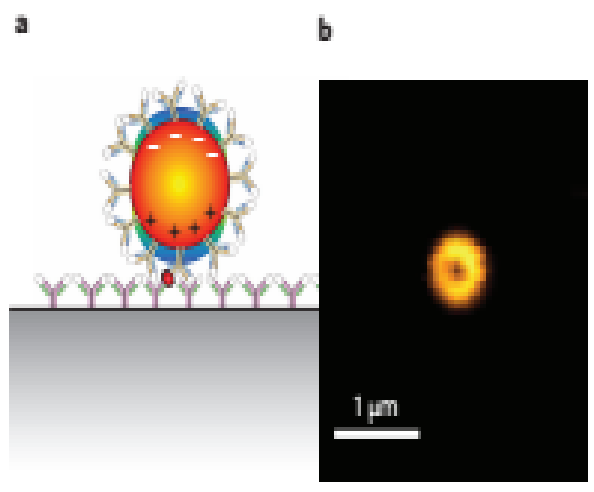

c

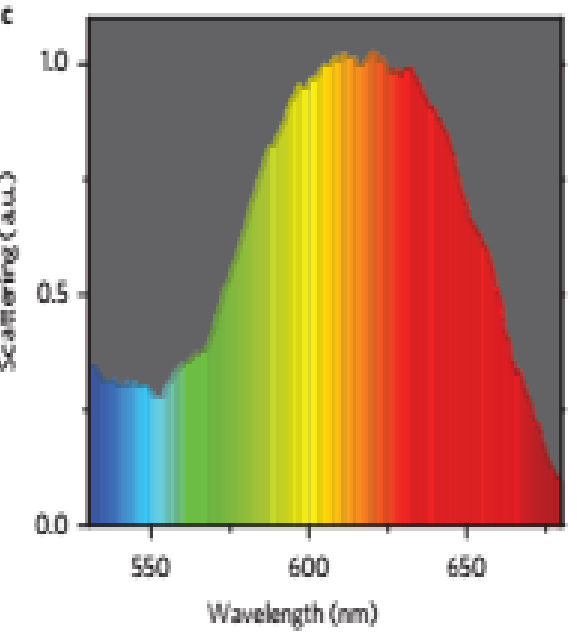

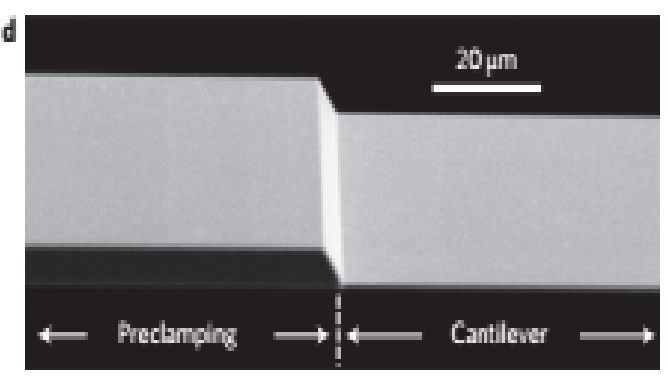
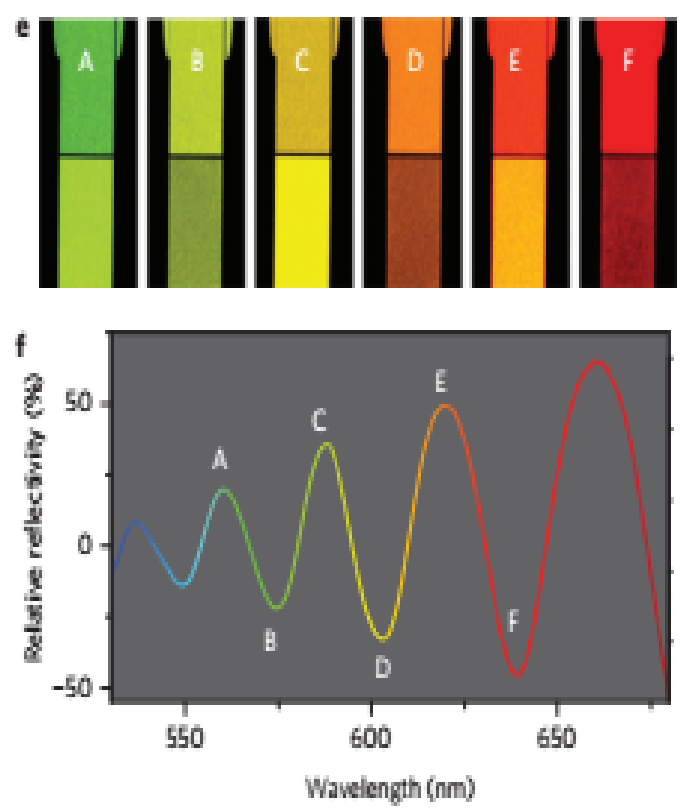

Figure 5: (A) The Au nanoparticles in the sandwich assay feature plasmon resonances that give rise to enhanced scattering and absorption near the resonance frequency. (B) The optical dark-field image of a single nanoparticle $100 \mathrm{~nm}$ in diameter after performing a sandwich assay. The doughnut shape is due to the resonance plasmon dipole that is perpendicular to the surface that dominates the scattering. (C) Scattering spectra of an area of $40 \mu \mathrm{m}$ in diameter that contains a single nanoparticle. (D) SEM image of the cantilever clamping region that indicates the frontier between the 6- $\mu \mathrm{m}$-thick preclamping structures fixed to the chip and the $1-\mu \mathrm{m}$-thick cantilever. (E) Bright-field images of the same cantilever clamping region at different illumination wavelengths in the visible spectrum. The thinness of the cantilever means the light can bounce efficiently multiple times between the opposite cantilever sides, and this enhances optical reflectivity at wavelengths in which constructive interference occurs. Conversely, the reflectivity suppression is seen for wavelengths in which destructive interference occurs. ( $F$ ), the relative reflectivity in the cantilever on preclamping as a function of the wavelength (Reproduced with permission from Ref. [68]. Copyright @ Nature Publishing Group). 
Citation: Barizuddin S, Bok S, Gangopadhyay S (2016) Plasmonic Sensors for Disease Detection - A Review. J Nanomed Nanotechnol 7: 373. doi:10.4172/2157-7439.1000373

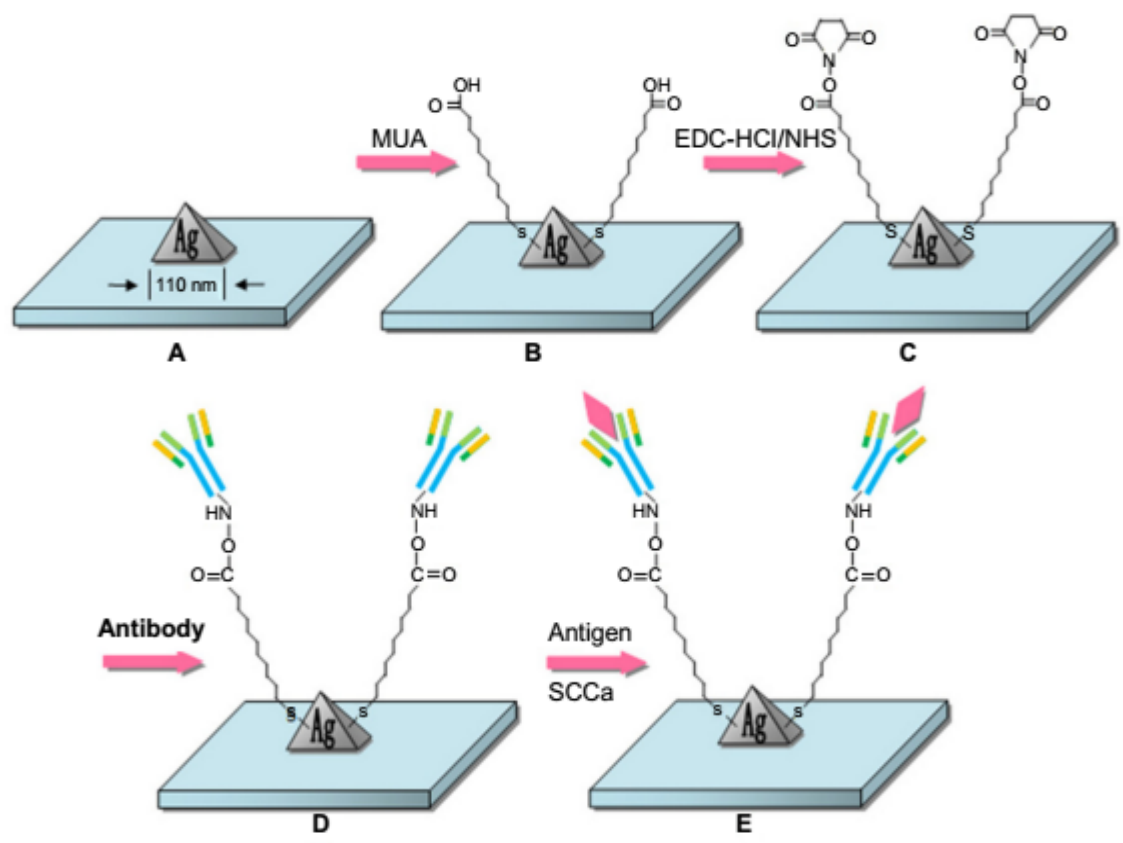

Figure 6: Design of the LSPR biosensor for specific detection of SCCa. (A) the glass substrate with triangle-shaped silver nanoparticles arrays. (B) saM formed due to the incubation of $1 \mathrm{mM} \mathrm{MUa}$. (C) Incubation in $75 \mathrm{mM} \mathrm{eDc-hcl} / 15 \mathrm{mM} \mathrm{Nhs}$. (D) attachment of anti-scca antibody (10 $\mu \mathrm{g} / \mathrm{ml})$. (E) The immunoassay of SCCa in both buffer and serum samples. (Copyright $@ 2014$ Zhao et al. This work was published by Dove Medical Press Limited, and licensed under Creative Commons Attribution - Non Commercial (unported, v3.0) License) [79].

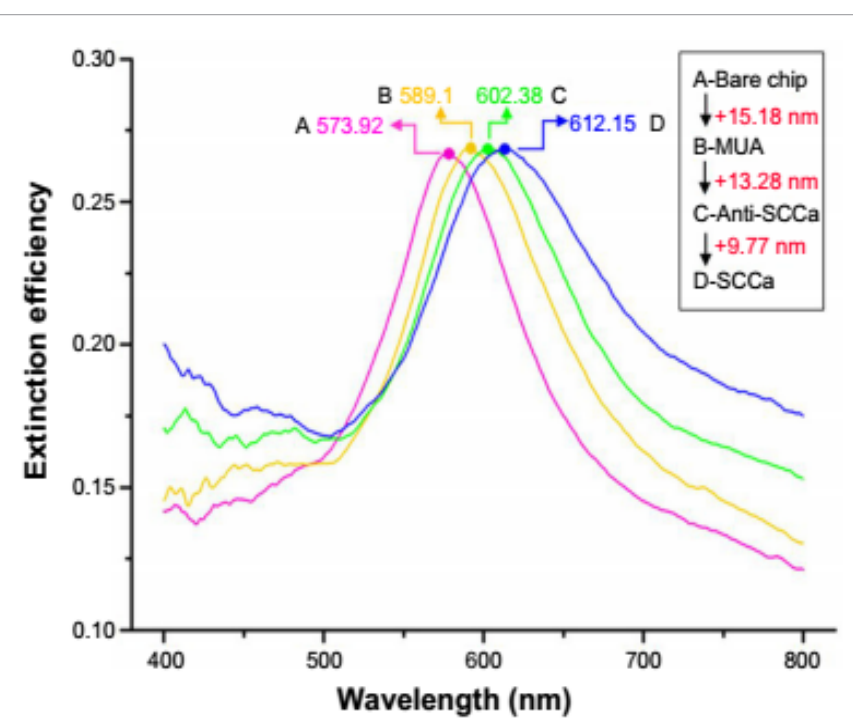

Figure 7: LSPR spectra for detection of $100 \mathrm{pM}$ scca. (A) Bare silver nanochip, $\lambda \max =573.92 \mathrm{~nm}$. (B) Modification of $1 \mathrm{mM} \mathrm{MUa}, \lambda \max =589.1 \mathrm{~nm}$. (C) Incubation with $10 \mu \mathrm{g} / \mathrm{ml}$ monoclonal anti-scca, $\lambda \max =602.38 \mathrm{~nm}$. (D) Detection of $100 \mathrm{pm}$ scca, $\lambda$ max $=612.15 \mathrm{~nm}$. (Copyright (C) 2014 Zhao et al. This work was published by Dove Medical Press Limited, and licensed under Creative Commons Attribution - Non Commercial (unported, v3.0) License) [79].

to yield a relatively small shift in the LSPR peak. Therefore, it is necessary to have an absorbance spectroscopy setup to enable detection. This analysis could be performed by a plate reader. For direct identification of physiological fluids that require transmission of electromagnetic radiation, it is advantageous to use the near-infrared region of the spectrum where light penetration is at its optimum. This means working in the 700-900 $\mathrm{nm}$ area of the light spectrum. The quantification of hepatitis B surface antigen (HBsAg) in blood serum and plasma, which indicates active viral replication of hepatitis B virus is shown in Figure 8. The LSPR shift occurs in the 700-750 nm, which allowed sensitive detection in physiological solutions. The HBsAg was detected using a monoclonal antibody conjugated to the surface of AuNRs by physical adsorption, and the surface was blocked using BSA. In pure buffers, the AuNR biosensor could measure the HBsAg concentration to a LOD of $0.01 \mathrm{IU} \mathrm{mL}$, which was 40 times lower than thee LOD of the ELISA.

\section{Detection of troponin (cTnI) for myocardial infarction}

The degree of peak shift observed analyte capture is proportional to the molecular weight and refractive index of the captured molecule. A uNR assay was used for the detection of cardiac troponin biomarker(cTnI) [82], an important biomarker of myocardial damage. Magnetic nanoparticles (MNPs, $\mathrm{Fe}_{3} \mathrm{O}_{4}$ ) were used to enhance the LSPR shift upon analyte binding (Figure 9). The high refractive index and mass of the iron oxide nanoparticles lead to a significant perturbation of LSPR when they are in close proximity to AuNRs. Furthermore, the high surface-to-volume ratio allows for a high density of chemical binding. The magnetic properties allow for the direct capture, separation and enrichment of target molecules in complex physiological conditions. In this assay, two different anti-cTnI antibodies were employed that bound to different epitopes on the cTnI molecule. One of these antibodies was conjugated to the MNPs, the other to the AuNRs. The MNPs were added to blood plasma, after that they bound the target cTnI, followed by magnetic separation of the cTnI. The analyte solution and AuNR solutions were then combined, and the analyte bound to the antibody on the AuNR surface, thus bringing the MNP in proximity to the Au surface. A LOD of $30 \mathrm{pM}$ in blood plasma was obtained, which is three orders of magnitude lower than comparable studies. The average phase shift was $210 \%$ compared to the analyte. 
Citation: Barizuddin S, Bok S, Gangopadhyay S (2016) Plasmonic Sensors for Disease Detection - A Review. J Nanomed Nanotechnol 7: 373. doi:10.4172/2157-7439.1000373
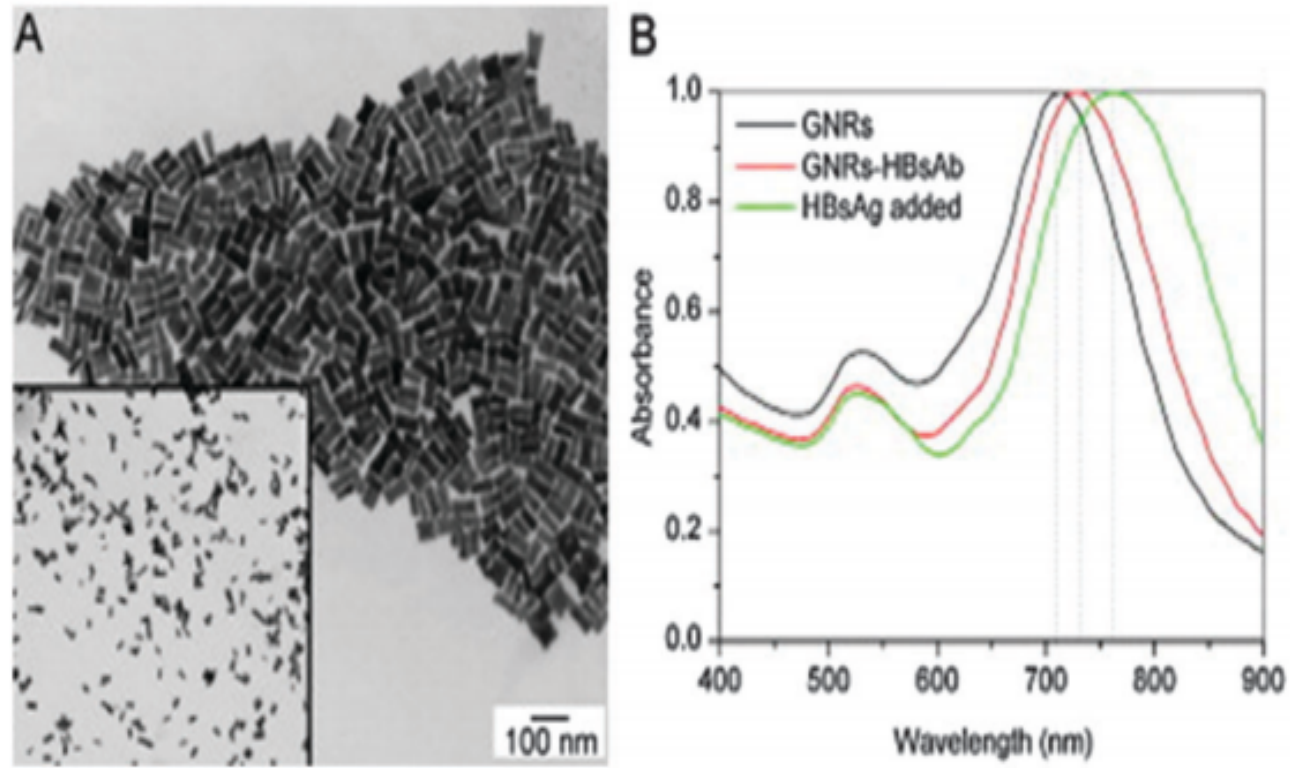

Figure 8: (A) TEM image of AuNRs used to detect hepatitis B surface antigen (HBsAg). (B) Absorbance spectrum, showing a red shift in the spectrum on the addition of the analyte. (Adapted from Ref. [82] and reprinted with permission from Elsevier).

\section{High efficiency extraction of cTnl by functionalized MNPs}

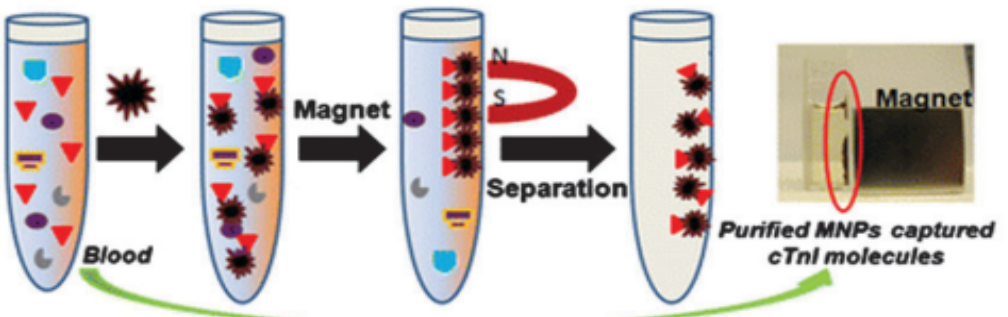

MNPs captured cTnI rendered to LSPR assay

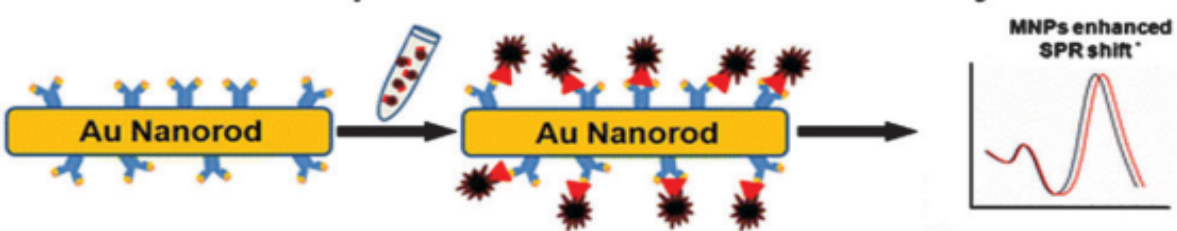

$*$ MNP conjugated anti-cTnl $::$ \} Non-specific biomolecules
$-\mathrm{cTnl}$ Capture anti-cTnl on Au nanorods

Figure 9: A schematic illustration of the bio-separation of target molecules from blood plasma using functional Fe $\mathrm{O}_{4}$ MNPs, followed by the MNP mediated LSPR assay. The use of MNPs facilitates the enhancement of the LSPR shift. (Adapted from Ref. [82], and reprinted with permission from The American Chemical Society).

\section{Detection of mtb antigens for tuberculosis}

Tuberculosis (TB) is an infectious disease that causes millions of deaths annually. The conventional "culture" methods require up to 6 to 8 weeks to provide a result. A SPR biosensor based on an array format shown in Figure 10 [83-86] allows immobilization of nine $\mathrm{TB}$ antigens onto the sensor chip for simultaneous determination of antibodies. Twenty-five-spot protein arrays were fabricated using conventional photolithography techniques. The array dimensions were $2 \mathrm{~mm}$ in diameter with a center-to-center spacing of $3 \mathrm{~mm}$. The 8-mercaptooctanoic (8-MOA) acid in ethanol was dropped on the surface array and allowed to form a self-assembled monolayer (SAM) for $30 \mathrm{~min}$. The surface of the array was further treated with $400 \mathrm{mM}$ 1-ethyl-3-(3-dimethylaminopropyl) carbodiimide hydrochloride) EDC/100 mM NHS for 10 minutes to active carboxyl groups of 8-MOA. A concentration of $50 \mu \mathrm{g} / \mathrm{mL}$ was maintained for all antigens and exposed to an array of protein spots for one hour. The arrays were blocked with $1 \mathrm{M}$ ethanolamine for $10 \mathrm{~min}$. The images shown were observed at the fixed angle which was slightly less than the SPR angle and collected with a CCD camera at a wavelength of $790 \mathrm{~nm}$. 
Citation: Barizuddin S, Bok S, Gangopadhyay S (2016) Plasmonic Sensors for Disease Detection - A Review. J Nanomed Nanotechnol 7: 373. doi:10.4172/2157-7439.1000373

Page 8 of 10

The Figure 11 spectra of the multiplexed detection system show the real-time SPR binding curves of the sample with PBS as the running buffer [83]. The change in reflectivity indicates an interaction between the specific antibody with its antigen in the serum. In this study, the detection signal reflectivity was shown to be at least two orders-ofmagnitude higher than in the negative control [87].

\section{Detection of human epidermal receptor protein-2 (HER2) for breast cancer}

Though SPR has been used for biomolecular detection of tumor biomarkers, it presents some experimental difficulties that were overcome by incorporating nano-hole arrays in metallic films. This configuration exhibits superior optical transmission [88-90], which is used in the monitoring of bio interactions on the plasmonic metal surface. This biosensor exhibited good sensitivity and linearity. For the detection of the HER2 antigen, the surface was coated with AB1 (biotinylated antibody) through its interaction with previously immobilized streptavidin molecules. Then, the HER2 antigen was immobilized, and finally, the surface was introduced to the AB2 antigen. The use of a secondary antibody, which characterizes the sandwich detection mode, improved the sensitivity of the sensor. As the molecules are immobilized on the surface, the refractive index on the metal-dielectric interface increases, leading to a redshift. Compared to the AB1 immobilization step, a shift of $1.2 \mathrm{~nm}$ was observed when the HER2 [91-93] antigen (concentration $30 \mathrm{ng} \mathrm{mL}^{-1}$ ) was immobilized. An extra redshift of $1.3 \mathrm{~nm}$ was observed when $\mathrm{AB} 2$ was linked to the AB1/HER2 layer; this resulted in a $2.5-\mathrm{nm}$ total band displacement. Furthermore, the maximum transmission shift obtained for the immobilization of AB2 was of $2.50 \pm 0.03 \mathrm{~nm}$ (with respect to the surface covered with $A B 1$ ) with an accuracy of $0.1 \mathrm{~nm}$. A concentration of $30 \mathrm{ng} \mathrm{mL}^{-1}$ of human epidermal receptor protein-2 (HER2) antigen [91,92] associated with breast cancer was detected using this system.

\section{Conclusion}

In this review paper, we have tried to highlight the wide use of plasmonic-based sensors and systems for disease detection. The reason for plasmonic diagnostics to be expanding at this rapid pace is the capability for ultra-sensitive detection. Most of the current diagnostics are based on ELISA, PCR or imaging. Fluorescence-based ELISA was also limited in the detection of concentrations, and only with plasmonics
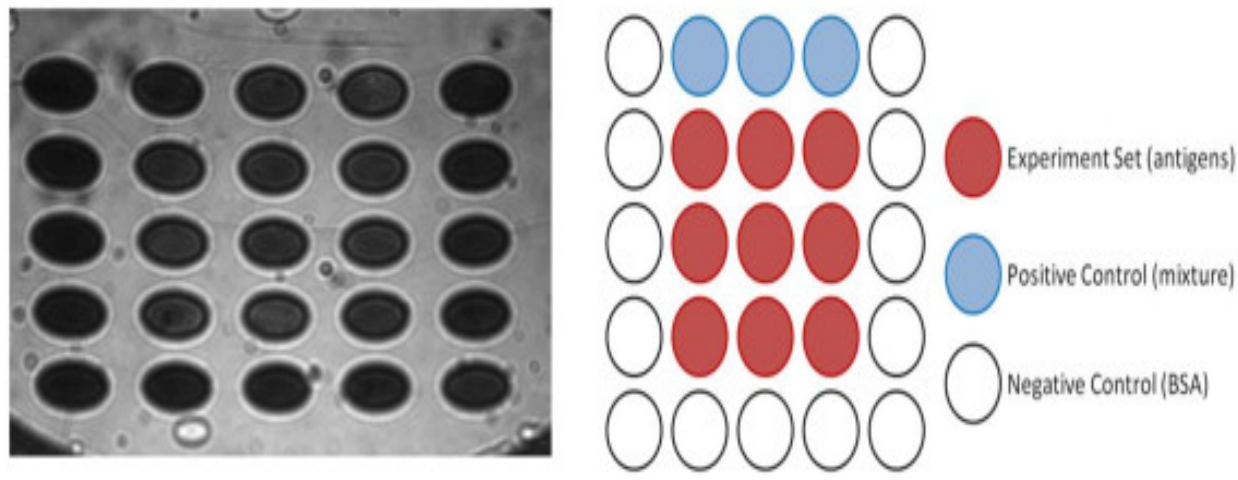

Figure 10: SPR image of a multiplexed microarray (left) and the biomolecule configuration on the array (right). (Copyright $\odot 2012$ Hsieh et al. licensee Springer [83]).

(a)

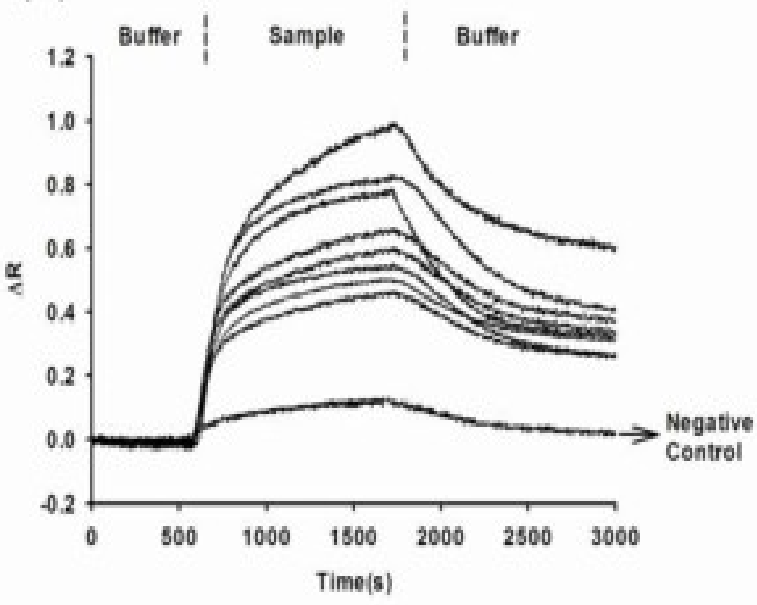

(b)

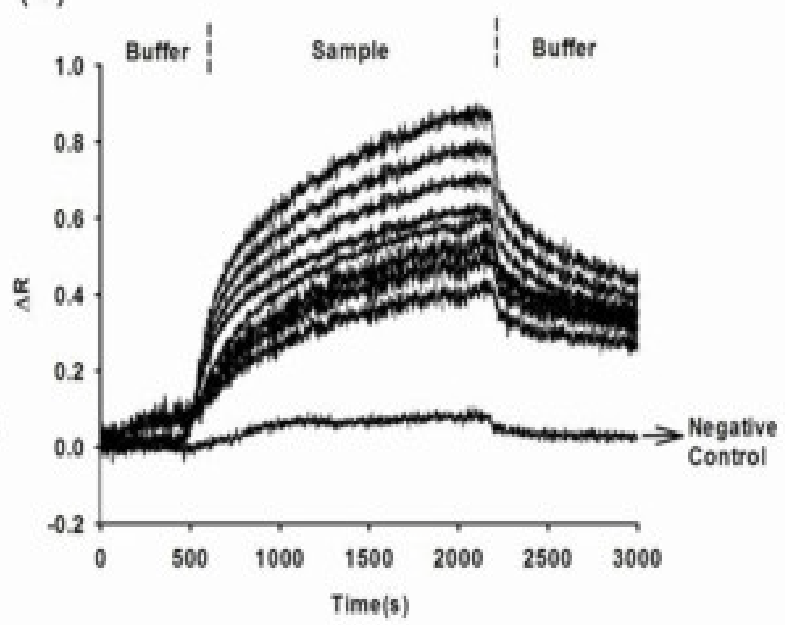

Figure 11: SPR sensograms shows a response to the injection of 10-fold diluted sera into an array-format SPR sensor. (a) SPR fromTB patient sera and (b) SPR results from normal control sera. (Copyright @2012 Hsieh et al. licensee Springer) [83]. 
was able to detect in femtogram $/ \mathrm{ml}$ or attogram $/ \mathrm{ml}$. Incorporating plasmonics methods along with older modalities of detection can result in complementary and synergicstic testing for disease states with improved sensitivity and specificity. Further advancements in the field of plasmonics could produce better diagnostic platforms and systems which may play an important role in prognosis, monitoring or detection of disease.

\section{References}

1. Université PS, Greffet (2012) Plasmonics. Plasmonics 167: 105-148.

2. Pelton M, Aizpurua J, Bryant G (2008) Metal-nanoparticle plasmonics. Laser Photonics Rev 2: 136-159.

3. Heber J (2009) Plasmonics: Surfing the wave. Nature 461: 720-722.

4. MacDonald KF, Zheludev NI (2010) Active plasmonics: Current status. Laser Photonics Rev 4: 562-567.

5. Gramotnev DK, Bozhevolnyi SI (2010) Plasmonics beyond the diffraction limit. Nat Photonics 4: 83-91.

6. Kerrn (2012) Perspective on plasmonics. Nat Photonics 6: 714-715.

7. Maier SA, Brongersma ML, Kik PG, Meltzer S, Requicha AAG, et al. (2001) Plasmonics - A route to nanoscale optical devices. Adv Mater 13: 1501-1505.

8. Fang $\mathrm{N}$, Lee $\mathrm{H}$, Sun $\mathrm{C}$, Zhang $X(2005)$ Sub-diffraction-limited optical imaging with a silver superlens. Science 308: 534-537.

9. Kawata S, Inouye Y, Verma P (2009) Plasmonics for near-field nano-imaging and superlensing. Nat Photonics 3: 388-394.

10. Zhang X, Liu Z (2008) Superlenses to overcome the diffraction limit. Nat Mater 7: 435-441.

11. Blaikie RJ (2006) Super-resolution imaging and performance optimisation for single- and multi-layer silver superlenses. Photonic Metamaterials From Random to Period.

12. Wang C, Astruc D (2014) Nanogold plasmonic photocatalysis for organic synthesis and clean energy conversion. Chem Soc Rev 43: 7188-7216.

13. Desai AS, Chauhan VM, Johnston APR, Esler T, Aylott JW (2014) Fluorescent nanosensors for intracellular measurements: Synthesis, characterization, calibration, and measurement. Front Physiol.

14. Lu AH, Salabas EL, Schüth F (2007) Magnetic nanoparticles: synthesis, protection, functionalization, and application. Angew Chem Int Ed Engl 46: 1222-1244.

15. Sun S, Zeng H (2002) Size-controlled synthesis of magnetite nanoparticles. J Am Chem Soc 124: 8204-8205.

16. Thakkar KN, Mhatre SS, Parikh RY (2010), Biological synthesis of metallic nanoparticles. Nanomedicine Nanotechnology Biol Med 6: 257-262.

17. Narayanan KB, Sakthivel N (2010) Biological synthesis of metal nanoparticles by microbes. Adv Colloid Interface Sci 156: 1-13.

18. Li N, Tittl A, Yue S, Giessen H, Song C, et al. (2014) DNA-assembled bimetallic plasmonic nanosensors. Light Sci Appl 3: e226.

19. Klimov VV, Guzatov DV (2007) Plasmonic atoms and plasmonic molecules. Appl Phys A Mater Sci Process 89: 305-314.

20. Boriskina SV, Ghasemi H, Chen G (2013) Plasmonic materials for energy: From physics to applications. Mater Today 16: 375-386.

21. Jain PK, El-Sayed MA (2010) Plasmonic coupling in noble metal nanostructures. Chem Phys Lett 487: 153-164.

22. Chen Z, Cui L, Song X, Yu L, Xiao J (2015) DNA-based plasmonic nanostructures. Opt Commun 340: 1-4.

23. Maksymov IS, Davoyan AR, Miroshnichenko AE, Simovski C, Belov $P$, et al (2012) Multifrequency tapered plasmonic nanoantennas. Opt Commun 285 821-824.

24. Rossouw D, Botton GA (2012) Resonant optical excitations in complementary plasmonic nanostructures. Opt Express 20: 6968-6973.

25. Fan JA, Bao K, Sun L, Bao J, Manoharan VN, et al. (2012) Plasmonic mode engineering with templated self-assembled nanoclusters. Nano Lett 12: 53185324.
26. Anker JN, Hall WP, Lambert MP, Velasco PT, Mrksich M, et al. (2009) Detection and Identification of Bioanalytes with High Resolution LSPR Spectroscopy and MALDI Mass Spectrometry. J Phys Chem C Nanomater Interfaces 113: 5891 5894.

27. Wu C, Xu QH (2009) Stable and functionable mesoporous silica-coated gold nanorods as sensitive localized surface plasmon resonance (LSPR) nanosensors. Langmuir 25: 9441-9446.

28. Sepúlveda B, Angelomé PC, Lechuga LM, Liz-Marzán LM (2009) LSPR-based nanobiosensors. Nano Today 4: 244-251.

29. Yang Z, Chen S, Fang P, Ren B, Girault HH, et al. (2013) LSPR properties of metal nanoparticles adsorbed at a liquid-liquid interface. Phys Chem Chem Phys 15: 5374-5378.

30. Hall WP, Ngatia SN, Van Duyne RP (2011) LSPR Biosensor Signa Enhancement Using Nanoparticle-Antibody Conjugates. J Phys Chem C Nanomater Interfaces 115: 1410-1414.

31. Haes AJ, Haynes CL, McFarland AD, Schatz GC, Van Duyne RP, et al. (2005) Plasmonic Materials for Surface-Enhanced Sensing and Spectroscopy. MRS Bull 30: 368-375.

32. Liu Z, Steele JM, Srituravanich W, Pikus Y, Sun C, et al. (2005) Focusing surface plasmons with a plasmonic lens. Nano Lett 5: 1726-1729.

33. Kumar GVP (2012) Plasmonic nano-architectures for surface enhanced Raman scattering: a review. J Nanophotonics 6: 064503.

34. Yonzon CR, Zhang X, Zhao J, Van Duyne RP (2007) Surface-Enhanced Nanosensors. Spectroscopy 22: 42

35. Klar TA (2007) Biosensing with plasmonic nanoparticles, in: Nanophotonics with Surf. Plasmons.

36. Tabor C, Van Haute D, El-Sayed MA (2009) Effect of orientation on plasmonic coupling between gold nanorods. ACS Nano 3: 3670-3678.

37. Hoessbacher C, Fedoryshyn Y, Emboras A, Melikyan A, Kohl M, Hillerkuss D et al. (2014) The plasmonic memristor: a latching optical switch. Optica 1: 198

38. Min C, Veronis G (2009) All-optical absorption switches in subwavelength metal-dielectric-metal plasmonic waveguides. Nano 1: 3-4.

39. Evgrafov A, Rupp CJ, Dunn ML, Maute K (2008) Optimal synthesis of tunable elastic wave-guides. Comput Methods Appl Mech Eng 198: 292-301.

40. Gather MC, Meerholz K, Danz N, Leosson K (2010) Net optical gain in a plasmonic waveguide embedded in a fluorescent polymer. Nat Photonics 4 457-461.

41. Hulteen JC, Van Duyne RP (1995) Nanosphere lithography: A materials genera fabrication process for periodic particle array surfaces. J Vac Sci Technol A Vacuum, Surfaces, Film 13: 1553.

42. Barbillon G (2012) Plasmonic nanostructures prepared by soft UV nanoimprint lithography and their application in biological sensing. Micromachines 3: 21-27.

43. Foulkes JE, Blaikie RJ (2011) Performance enhancements to absorbancemodulation optical lithography. II. Plasmonic superlenses. J Opt Soc Am A Op Image Sci Vis 28: 2218-2225.

44. Guillot N, de la Chapelle ML (2012) Lithographied nanostructures as nanosensors. J Nanophotonics 6: 064506-1.

45. Zhou W, Dridi M, Suh JY, Kim CH, Co DT, et al. (2013) Lasing action in strongly coupled plasmonic nanocavity arrays. Nat Nanotechnol 8: 506-511.

46. Bansal A, Sekhon JS, Verma SS (2014) Scattering Efficiency and LSPR Tunability of Bimetallic Ag, Au, and Cu Nanoparticles. Plasmonics 9: 143-150.

47. Kern AM, Martin OJ (2011) Excitation and reemission of molecules near realistic plasmonic nanostructures. Nano Lett 11: 482-487.

48. Knight MW, King NS, Liu L, Everitt HO, Nordlander P, et al. (2014) Aluminum for plasmonics. ACS Nano 8: 834-840.

49. Srituravanich W, Fang N, Sun C, Luo Q, Zhang X (2004) Plasmonic nanolithography. Nano Lett 4: 1085-1088.

50. Matveeva EG, Gryczynski I, Barnett A, Leonenko Z, Lakowicz JR, et al. (2007) Metal particle-enhanced fluorescent immunoassays on metal mirrors. Ana Biochem 363: 239-245.

51. Gjonaj B, Aulbach J, Johnson PM, Mosk AP, Kuipers L, Lagendijk A, et al (2011) Active spatial control of plasmonic fields. Nat Photonics 5: 360-363. 
52. Shafiei F, Wu C, Wu Y, Khanikaev AB, Putzke P, Singh A, et al. (2013) Plasmonic nano-protractor based on polarization spectro-tomography. Nat Photonics 7: 367-372.

53. Wujcik EK, Wei H, Zhang X, Guo J, Yan X, Sutrave N, et al. (2014) Antibody nanosensors: a detailed review. RSC Adv 4: 43725-43745.

54. Li X, Jiang L, Zhan Q, Qian J, He S (2009) Localized surface plasmon resonance (LSPR) of polyelectrolyte-functionalized gold-nanoparticles for biosensing. Colloids Surfaces A Physicochem Eng Asp 332: 172-179.

55. Xu Z, Hou Y, Sun S (2007) Magnetic core/shell Fe3O4/Au and Fe3O4/Au/Ag nanoparticles with tunable plasmonic properties. J Am Chem Soc 129: 86988699.

56. Toma M, Toma K, Michioka K, Ikezoe Y, Obara D, et al. (2011) Collective plasmon modes excited on a silver nanoparticle 2D crystalline sheet. Phys Chem Chem Phys 13: 7459-7466.

57. Tabatabaei M, Najiminaini M, Davieau K, Kaminska B, Singh MR, Carson JJL, et al. (2015) Tunable 3D Plasmonic Cavity Nanosensors for Surface-Enhanced Raman Spectroscopy with Sub-femtomolar Limit of Detection. ACS Photonics 2: $752-759$.

58. Anker JN, Hall WP, Lyandres O, Shah NC, Zhao J, et al. (2008) Biosensing with plasmonic nanosensors. Nat Mater 7: 442-453

59. Raphael MP, Christodoulides JA, Mulvaney SP, Miller MM, Long JP, et al (2012) A new methodology for quantitative LSPR biosensing and imaging. Anal Chem 84: 1367-1373

60. Cash KJ, Clark HA (2012) In vivo histamine optical nanosensors. Sensors (Basel) 12: 11922-11932.

61. Bhatta D, Stadden E, Hashem E, Sparrow IJ, Emmerson GD (2010) Label-free monitoring of antibody-antigen interactions using optical microchip biosensors. $\mathrm{J}$ Immunol Methods 362: 121-126.

62. Mortazavi D, Kouzani AZ, Kaynak A, Duan W (2011) Nano-plasmonic biosensors: A review. IEEE/ICME Int Conf Complex Med Eng C 20: 31-36.

63. Li CY, Meng M, Huang SC, Li L, Huang SR, et al. (2015) "Smart" Ag Nanostructures for Plasmon-Enhanced Spectroscopies. J Am Chem Soc 137: 13784-13787.

64. Aćimović SS, Ortega MA, Sanz V, Berthelot J, Garcia-Cordero JL, et al. (2014) LSPR chip for parallel, rapid, and sensitive detection of cancer markers in serum. Nano Lett 14: 2636-2641.

65. Das S, Turunen J (2012) Plasmonic nanosensors in the treatment of cancer: an attempt to conquer the immortal illness. Inanophotonics IV.

66. Huang X, Jain PK, El-Sayed IH, El-Sayed MA (2008) Plasmonic photothermal therapy (PPTT) using gold nanoparticles. Lasers Med Sci 23: 217-228.

67. Volkert AA, Haes AJ (2014) Advancements in nanosensors using plastic antibodies. Analyst 139: 21-31.

68. Kosaka PM, Pini V, Ruz JJ, da Silva RA2, González MU, et al. (2014) Detection of cancer biomarkers in serum using a hybrid mechanical and optoplasmonic nanosensor. Nat Nanotechnol 9: 1047-1053.

69. Maier SA (2005) Plasmonics - Towards Subwavelength Optical Devices. Curr Nanosci 1: 17-22.

70. Gather C (2012) A rocky road to plasmonic lasers. Nat Photonics 6: 708

71. Jang MS, Atwater H (2011) Plasmonic rainbow trapping structures for light localization and spectrum splitting. Phys Rev Lett 107: 207401.

72. Guo L, Kim DH (2012) LSPR biomolecular assay with high sensitivity induced by aptamer-antigen-antibody sandwich complex. Biosens Bioelectron 31: 567-570.

73. Kim HM, Jin SM, Lee SK, Kim MG, Shin YB (2009) Detection of Biomolecular Binding Through Enhancement of Localized Surface Plasmon Resonance (LSPR) by Gold Nanoparticles. Sensors (Basel) 9: 2334-2344.

74. Vo-Dinh T, Cullum BM, Stokes DL (2001) Nanosensors and biochips: Frontiers in biomolecular diagnostics. Sensors Actuators B Chem 74: 2-11.

75. Wang J1 (2009) Biomolecule-functionalized nanowires: from nanosensors to nanocarriers. Chemphyschem 10: 1748-1755.

76. Hanske C, Tebbe M, Kuttner C, Bieber V, Tsukruk VV, et al. (2014) Strongly coupled plasmonic modes on macroscopic areas via template-assisted colloidal self-assembly. Nano Lett 14: 6863-6871.
77. Ji A, Sharma RP, Kumari A, Pathak NK (2014) Numerical simulation of solar cell plasmonics for small and large metal nano clusters using discrete dipole approximation. Plasmonics 9: 291-297.

78. Wang L, Nishijima Y, Ueno K, Misawa H, Tamai N (2012) Effect of dipole coupling on near-IR LSPR and coherent phonon vibration of periodic gold pair nanocuboids. J Phys Chem C 116: 17838-17846.

79. Qianying Zhao MX, Duan R, Yuan J, Quan Y, Yang H (2014) A reusable localized surface plasmon resonance biosensor for quantitative detection of serum squamous cell carcinoma antigen in cervical cancer patients based on silver nanoparticles array. Int J Nanomedicine 9 1097-1104

80. Xie L, Yan X, Du Y (2014) An aptamer based wall-less LSPR array chip for label-free and high throughput detection of biomolecules. Biosens Bioelectron 53: $58-64$

81. Yonzon CR, Stuart DA, Zhang X, McFarland AD, Haynes CL, et al. (2005) Towards advanced chemical and biological nanosensors-An overview. Talanta 67: $438-448$

82. Howes PD, Rana S, Stevens MM (2014) Plasmonic nanomaterials for biodiagnostics. Chem Soc Rev 43: 3835-3853.

83. Shang-Chen Hsieh CW, Chang X, Lu C, Wei C, Lin C, et al. (2012) Hsin-Chih Lai, Rapid identification of Mycobacterium tuberculosis infection by a new array format-based surface plasmon resonance method. Nanoscale Res Lett 7:180.

84. Silvestri D, Sonato A, Ruffato G, Meneghello A, Antognoli A, et al. (2015) A peptide nucleic acid label-free biosensor for Mycobacterium tuberculosis DNA detection via azimuthally controlled grating-coupled SPR. Anal Methods 7 : 4173-4180.

85. Prabhakar N, Arora K, Arya SK, Solanki PR, Iwamoto M, et al. (2008) Nucleic acid sensor for M. tuberculosis detection based on surface plasmon resonance. Analyst 133: 1587-1592.

86. Dallaire AM, Patskovsky S, Vall A, lisle MM (2015) Electrochemical plasmonic sensing system for highly selective multiplexed detection of biomolecules based on redox nanoswitches. Biosens Bioelectron 71: 75-81.

87. Tokel O, Yildiz UH2, Inci F2, Durmus NG3, Ekiz OO4, et al. (2015) Portable microfluidic integrated plasmonic platform for pathogen detection. Sci Rep 5 9152

88. Zhou ZK, Peng XN, Yang ZJ, Zhang ZS, Li M, et al. (2011) Tuning gold nanorod-nanoparticle hybrids into plasmonic Fano resonance for dramatically enhanced light emission and transmission. Nano Lett 11: 49-55

89. Wang Y, Srituravanich W, Sun C, Zhang X (2008) Plasmonic nearfield scanning probe with high transmission. Nano Lett 8: 3041-3045.

90. Chanda D, Shigeta K, Truong T, Lui E, Mihi A, et al. (2011) Coupling of plasmonic and optical cavity modes in quasi-three-dimensional plasmonic crystals. Nat Commun 2: 479

91. Peng G, Hakim M, Broza YY, Billan S, Abdah-Bortnyak R, et al. (2010) Detection of lung, breast, colorectal, and prostate cancers from exhaled breath using a single array of nanosensors. Br J Cancer 103: 542-551.

92. Monteiro JP, de Oliveira JH, Radovanovic E, Brolo AG, Girotto EM (2016) Microfluidic Plasmonic Biosensor for Breast Cancer Antigen Detection. Plasmonics 11: 45-51.

93. Hsin-Neng W, Tuan VD (2009) Multiplex detection of breast cancer biomarkers using plasmonic molecular sentinel nanoprobes. Nanotechnology 20: 6510165106. 